\title{
Risk factors of bronchopulmonary dysplasia in premature infants under 32 weeks of gestation: A retrospective case-control study
}

Li Yue ( $\nabla$ ly19871107@163.com )

Jiangsu Province Hospital and Nanjing Medical University First Affiliated Hospital

Shu Dong Cui

Jiangsu Province Hospital and Nanjing Medical University First Affiliated Hospital

\section{Research article}

Keywords: Risk factors of bronchopulmonary, dysplasia in premature infants under 32 weeks of gestation, A retrospective case control study

Posted Date: February 28th, 2020

DOI: https://doi.org/10.21203/rs.2.24799/v1

License: (c) (i) This work is licensed under a Creative Commons Attribution 4.0 International License.

Read Full License 
Risk factors of bronchopulmonary dysplasia in premature infants under 32 weeks of gestation: A retrospective case-control study

Yue Li, Shu Dong Cui*

* Correspondence: csd9758@126.com

Department of Pediatrics, First Affiliated Hospital of Nanjing Medical University, Nanjing 210000, Jiangsu Province, China

\begin{abstract}
Background: Bronchopulmonary dysplasia (BPD) in preterm infants is one of the most serious complications in neonatology, as it significantly affects the quality of life of preterm children. We retrospectively analyze the incidence and risk factors of BPD in premature infants under 32 weeks of gestation age.

Methods: Clinical data of premature infants with gestational age $\leq 32$ weeks admitted to the neonatal intensive care unit of the First Affiliated Hospital of Nanjing Medical University from 2015 to 2017 were selected for this retrospective case-control study. The patients were classified into the BPD and control groups based on the clinical diagnosis. We compared the general condition, treatments, complications, and clinical outcome of premature infants in both groups. Student's $t$-test, non-parametric test, and binary logistic regression were used to analyze the occurrence of
\end{abstract}


BPD and its potential risk factors.

Results: A total of 213 premature infants aged $\leq 32$ gestational weeks were included in the study; of these, $81(38 \%)$ were diagnosed with BPD. The gestational age $(29.14 \pm 1.88$ weeks vs. $30.82 \pm 0.98$ weeks, $\mathrm{P}<0.001)$ and weight $(1240 \pm 314.87 \mathrm{~g}$ vs. $1584.77 \pm 318.18 \mathrm{~g}, \mathrm{P}<0.001)$ in the BPD group were lower than those in the control group. The liquid quantity, urine volume, and calorie intake of preterm infants in the BPD group within one week after birth were significantly lower than those of infants in the control group. Logistic regression analysis showed that invasive mechanical ventilation $(\mathrm{OR}=5.065,95 \% \mathrm{CI}: 1.680-15.266)$; use of erythrocyte suspension $(\mathrm{OR}=10.146,95 \%$ CI: $1.860-55.336)$; and PCO2 in early postnatal arterial blood gas (OR=1.122, 95\% CI: $1.024-1.229)$ were risk factors for BPD. On the other hand, gestational age $(\mathrm{OR}=0.657$, 95\% CI: 0.460-0.937); calorie intake (OR=0.917, 95\% CI: 0.874-0.963); $\mathrm{PO} 2(\mathrm{OR}=0.925,95 \% \mathrm{CI}: 0.870-0.985)$; and $\mathrm{pH}$ in early postnatal arterial blood gas $(\mathrm{OR}<0.001,95 \%$ CI: $0.000-0.156)$ were protective factors for BPD.

Conclusion: The incidence of BPD in very low and extremely low birth weight infants is high. Maintaining early internal environment stability, increasing early calorie intake, shortening invasive mechanical ventilation time, reducing blood transfusion, and closing patent ductus arteriosus could likely reduce the incidence of BPD. 


\section{Background}

Bronchopulmonary dysplasia (BPD) in preterm infants is one of the most serious complications in neonatology, as it significantly affects the quality of life of preterm children. In China, the incidence of BPD gradually increased with the improvement of the treatment success rate of very low birth weight (VLBW) and extremely low birth weight (ELBW) infants. Reiterer [1] showed that the incidence of moderate and severe BPD in a single-center study in Austria was about 25\%. The incidence of BPD in premature infants aged $\leq 32$ gestational weeks is about $35 \%$, while this incidence among premature infants aged $<28$ gestational weeks can be as high as $80 \%$ [2]. While BPD is an independent risk factor for long-term dysplasia of the nervous system [3], effective treatment methods are still lacking. Therefore, prevention of BPD has become an area of clinical research focus. In this retrospective case-control study, we analyzed the occurrence and risk factors of BPD in preterm infants aged $\leq 32$ gestational weeks, to provide a clinical basis for the prevention of BPD.

\section{Methods}

Study Subjects

In this retrospective case-control study, a total of 213 premature infants with gestational age $\leq 32$ weeks and hospitalization duration $\geq 28$ days were selected from the neonatal intensive care unit (NICU) of the First 
Affiliated Hospital of Nanjing Medical University from 2015 to 2017. The exclusion criteria were severe congenital malformation, congenital heart diseases, inherited metabolic diseases, chromosomal disease, death, and treatment abandonment or refusal to provide informed consent by parents. All included patients' parents provided written informed consent. All procedures were conducted in accordance with the principles of the Declaration of Helsinki.

Patients were classified into the BPD and control groups according to the diagnosis of NICHD/NHLBI/ORD workshop [4]. Premature infants $\leq 32$ weeks still need oxygen inhalation until 36 weeks of gestational age or discharge, whichever comes first, and the required oxygen concentration is between $21 \%$ and $30 \%$ to diagnose moderate BPD. Severe BPD is diagnosed if the required oxygen concentration is $>30 \%$ and/or shows positive pressure. We compared the general condition, treatments, complications, and clinical outcome of premature infants in both groups. In this study, SPSS 22.0 software was used for statistical analysis. The categorical data were presented as actual numbers or percentages (\%), and the groups were compared by the $\chi^{2}$ test. Normally distributed data were compared using the $t$-test and presented as mean $\pm \mathrm{SD}$, whereas non-normally distributed data were compared after logarithmic transformation. Furthermore, correlative factor analysis of BPD was performed using logistic regression analysis. For all statistical tests, 
$\mathrm{P}<0.05$ was considered to indicate statistical significance.

\section{Results}

Of the 213 included preterm infants, 81 had BPD (BPD group); the remaining 132 infants comprised the control group. The overall incidence of BPD was 38\%: the proportion of mild BPD was $79.0 \%(n=64)$, and the proportion of moderate and severe BPD was $21.0 \%(n=17)$. The proportion of extremely low and ultra-low birth weight infants in the BPD group was $61.7 \%(n=50)$, significantly higher than that in the control group (33.3\% [ $\mathrm{n}=44], \mathrm{P}<0.001)$. Gestational age and weight showed significant intergroup differences $(\mathrm{P}<0.001)$, but sex did not $(\mathrm{P}=0.214)$ (Table 1).

Table 1 Comparison of hospitalization data between two groups of premature infants

\begin{tabular}{lllll}
\hline & control group & BPD group & $t$ or $\chi^{2}$ & P value \\
\hline gender & $70 / 62$ & $50 / 31$ & $1.544^{\mathrm{b}}$ & 0.214 \\
gestational age & $30.82 \pm 0.98$ & $29.14 \pm 1.88$ & $7.443^{\mathrm{a}}$ & $<0.001$ \\
birth weight & $1584.77 \pm 318.18$ & $1240 \pm 314.87$ & $7.691^{\mathrm{a}}$ & $<0.001$ \\
\hline
\end{tabular}

a: $t$ value of Student's $t$-test; b: $\chi^{2}$ value of $\chi^{2}$ test

The rates of use of invasive mechanical ventilation (54.3\% vs. $9.8 \%$, $\mathrm{P}<0.001)$ and non-invasive mechanical ventilation (92.6\% vs. $54.5 \%$, $\mathrm{P}<0.001)$ in the BPD group were higher than those in the control group. Similarly, the use time of both types of mechanical ventilation in the BPD group was higher than that in the control group $(\mathrm{P}<0.001)$ (Table 2).

Table 2 Comparison of the different types of mechanical ventilation in two groups of premature infants 


\begin{tabular}{|c|c|c|c|c|}
\hline & control group & BPD group & $t$ or $\chi^{2}$ & $P$ value \\
\hline invasive mechanical ventilation & $13 / 119$ & $44 / 37$ & $50.655^{\mathrm{c}}$ & $<0.001$ \\
\hline the time of invasive mechanical ventilation (day) ${ }^{\mathrm{a}}$ & $3.62 \pm 2.399$ & $3.57 \pm 5.069$ & $-2.914^{\mathrm{b}}$ & 0.006 \\
\hline non-invasive mechanical ventilation & $72 / 60$ & $75 / 6$ & $33.980^{\mathrm{c}}$ & $<0.001$ \\
\hline the time of non-invasive mechanical ventilation (day) ${ }^{a}$ & $3.32 \pm 2.07$ & $9.99 \pm 6.85$ & $-8.044^{\mathrm{b}}$ & $<0.001$ \\
\hline
\end{tabular}

a: abnormally distributed continuous variables were log-transformed for analysis, non-transformed values were displayed for ease of interpretation; $\mathrm{b}: t$ value of Student's $t$-test; $\mathrm{c}: \chi^{2}$ value of $\chi^{2}$ test

The proportion of preterm infants in the BPD group requiring plasma

(77.8\% vs. $29.5 \%, \mathrm{P}<0.001)$; erythrocyte suspension (50.6\% vs. $27.3 \%$, $\mathrm{P}=0.001)$; and gamma globulin $(18.5 \%$ vs. $2.3 \%, \mathrm{P}<0.001)$ was higher than that in the control group, but there was no significant intergroup difference in the mean volume of plasma and gamma globulin required. The mean volume of erythrocyte suspension in the BPD group was higher than that in the control group $(45.83 \pm 14.72 \mathrm{~mL}$ vs. $37.56 \pm 15.03 \mathrm{~mL}$, $\mathrm{P}=0.001$ ) (Table 3).

Table 3 Comparison of the use of blood products in two groups of premature infants

\begin{tabular}{lllll}
\hline & control group & BPD group & $t$ or $\chi^{2}$ & P value \\
\hline plasma & $39 / 93$ & $63 / 18$ & $46.794^{\mathrm{c}}$ & $<0.001$ \\
the mean volume of plasma (ml) & $50.10 \pm 25.95$ & $52.69 \pm 38.43$ & $-0.407^{\mathrm{b}}$ & 0.685 \\
erythrocyte suspension & $36 / 96$ & $41 / 40$ & $11.852^{\mathrm{c}}$ & 0.001 \\
the mean volume of erythrocyte suspension $(\mathrm{ml})$ & $37.56 \pm 15.03$ & $45.83 \pm 14.72$ & $2.432^{\mathrm{b}}$ & 0.017 \\
gamma globulin & $3 / 129$ & $15 / 66$ & $17.124^{\mathrm{c}}$ & $<0.001$ \\
the mean volume of gamma globulin $(\mathrm{ml})^{\mathrm{a}}$ & $27.53 \pm 14.53$ & $30.67 \pm 17.21$ & $0.332^{\mathrm{b}}$ & 0.744 \\
\hline
\end{tabular}

a: the concentration of gamma globulin: $2.5 \mathrm{~g} / 50 \mathrm{ml}$; $\mathrm{b}: t$ value of Student's $t$-test; c: $\chi^{2}$ value of $\chi^{2}$ test

In the first week after birth, fluid and calorie intake of premature infants in the BPD group were significantly lower than those in the control group

(Figs. 1A, 1C). Further, urine volume in the BPD group was significantly 
lower than that in the control group from day 2 (Fig. 1B).
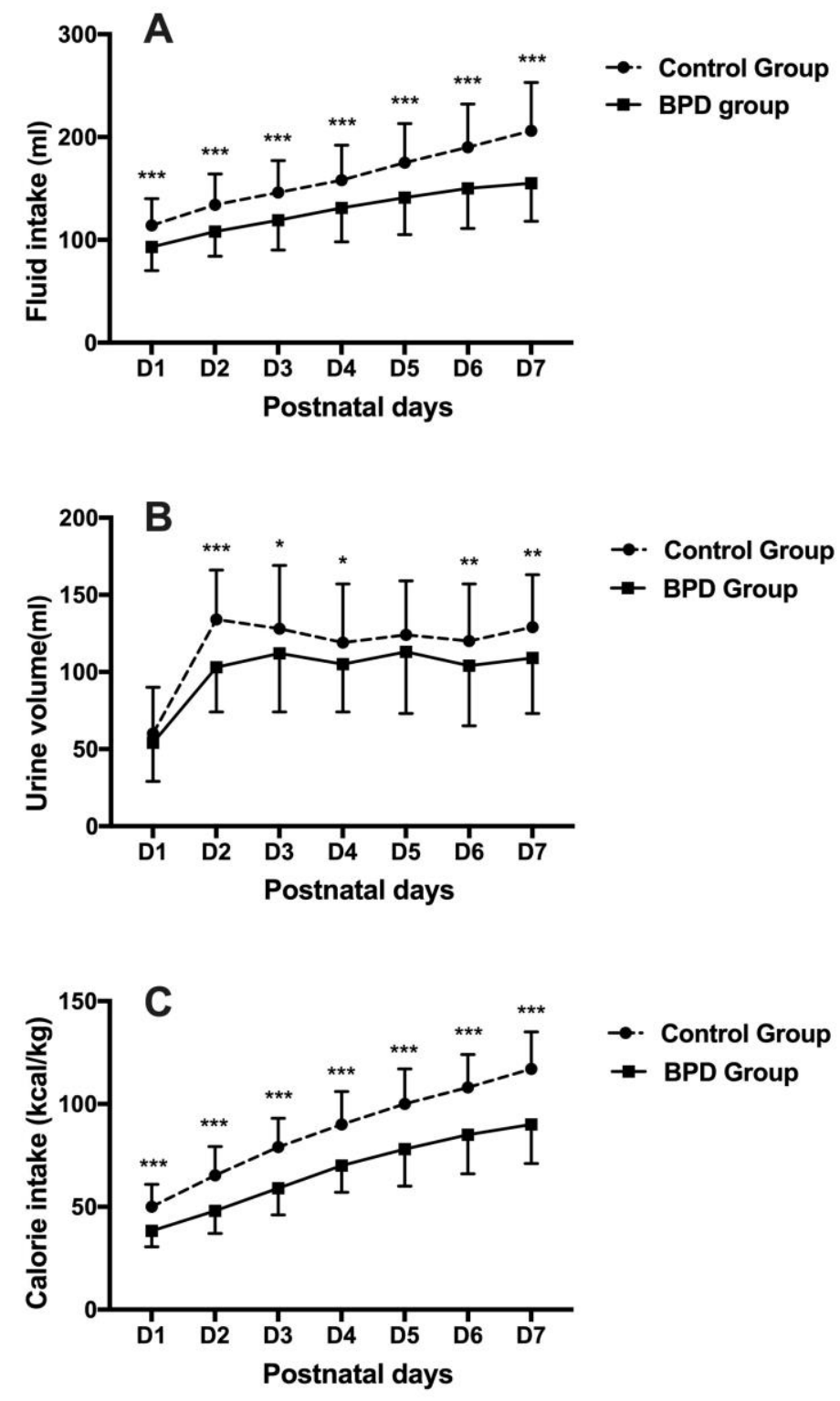

Fig. 1 Comparison of fluid intake, urine volume and calorie intake in two groups within one week after birth

$* \mathrm{P}<0.05, * * \mathrm{P}<0.01, * * * \mathrm{P}<0.001$

The blood gas analysis with the lowest daily PO2 monitoring was selected for analysis. The blood gas analysis showed that the $\mathrm{pH}$ value of the BPD group was lower than that of the control group on the first day after birth; however, there was no significant intergroup difference on the second, third, and seventh days after birth. Moreover, blood gas analysis 
on days 1, 2, 3, and 7 after delivery showed that the PCO2 and PO2 levels in the BPD group were higher and lower, respectively, than those in the control group. $\mathrm{HCO} 3$ of the BPD group was significantly higher than that of the control group on days 3 and 7, but there was no statistical difference between the two groups on days 1 and 2 (Fig. 2).
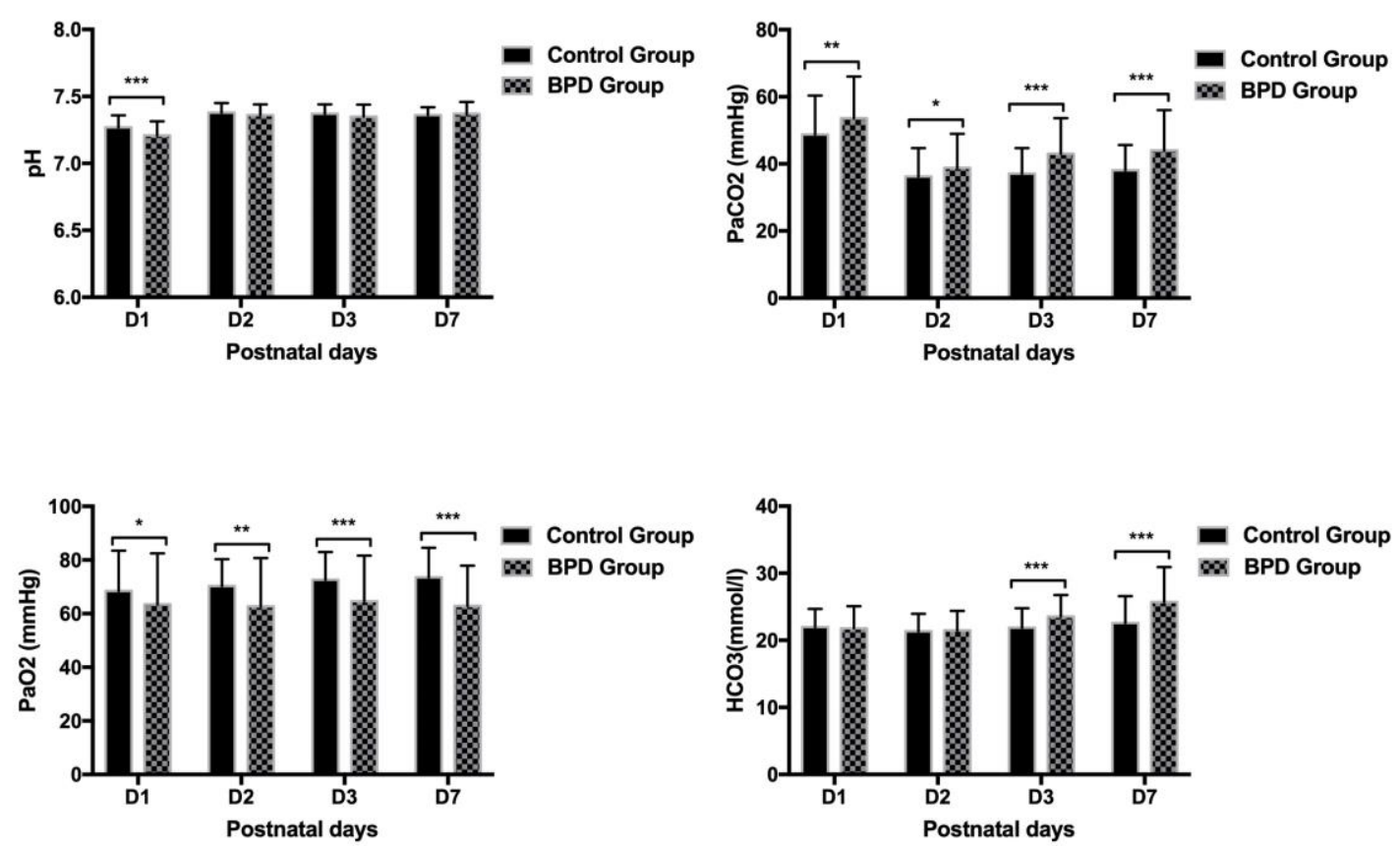

Fig. 2 Comparison of blood gas analysis in two groups within one week after birth $* \mathrm{P}<0.05, * * \mathrm{P}<0.01, * * * \mathrm{P}<0.001$

In our study, the proportion of respiratory distress syndrome (RDS) in the BPD group was $100 \%$, but not all babies born with RDS had BPD. The average grade of RDS in the BPD group was significantly higher than that in the control group $(2.58 \pm 0.68$ vs. $1.86 \pm 0.65, \mathrm{P}<0.001)$. The incidence of patent ductus arteriosus (PDA) in the BPD group (44.4\% vs. $23.5 \%, \mathrm{P}=0.001)$ and the size of the PDA $(3.09 \pm 1.28 \mathrm{~mm}$ vs. $2.51 \pm 0.89$ 
$\mathrm{mm}, \mathrm{P}=0.04$ ) were significantly higher than those in the control group.

However, there was no significant difference with regard to onset time of anemia between the two groups (Table 4).

Table 4 Comparison of complications in two groups of premature infants

\begin{tabular}{lllll}
\hline & control group & BPD group & $t$ or $\chi^{2}$ & P value \\
\hline RDS & $86 / 46$ & $81 / 0$ & $36.002^{\mathrm{c}}$ & $<0.001$ \\
the average grade of RDS & $1.86 \pm 0.65$ & $2.58 \pm 0.68$ & $-6.939^{\mathrm{b}}$ & $<0.001$ \\
PDA & $31 / 101$ & $36 / 45$ & $10.228^{\mathrm{c}}$ & 0.001 \\
the size of the PDA $^{\text {onset time of anemia (day) }}{ }^{\mathrm{a}}$ & $2.51 \pm 0.89$ & $3.09 \pm 1.28$ & $-3.077^{\mathrm{b}}$ & 0.040 \\
\hline
\end{tabular}

a: abnormally distributed continuous variables were log-transformed for analysis, non-transformed values were displayed for ease of interpretation; b: $t$ value of Student's $t$-test; $\mathrm{c}$ : $\chi^{2}$ value of $\chi^{2}$ test

Logistic regression analysis was conducted with the difference related factors as independent variables and the occurrence of BPD as the dependent variable. Our analysis showed that invasive mechanical ventilation, use of erythrocyte suspension, and PCO2 level in early postnatal arterial blood were risk factors for BPD, while gestational age, calorie intake, $\mathrm{PO} 2$, and $\mathrm{pH}$ were protective factors for BPD (Table 5).

Table 5 Logistic regression analysis of risk factors in BPD

\begin{tabular}{lllllll}
\hline & $\beta$ & standard error & Wald & P value & OR & $95 \% \mathrm{CI}$ \\
\hline gestational age & -0.421 & 0.182 & 5.364 & 0.021 & 0.657 & $0.460-0.937$ \\
invasive mechanical ventilation & 1.622 & 0.563 & 8.306 & 0.004 & 5.065 & $1.680-15.266$ \\
erythrocyte suspension & 2.317 & 0.866 & 7.167 & 0.007 & 10.146 & $1.860-55.336$ \\
calorie intake & -0.086 & 0.025 & 12.048 & 0.001 & 0.917 & $0.874-0.963$ \\
$\mathrm{PCO}_{2}$ & 0.115 & 0.046 & 6.112 & 0.013 & 1.122 & $1.024-1.229$ \\
$\mathrm{PO}_{2}$ & -0.077 & 0.032 & 5.971 & 0.015 & 0.925 & $0.870-0.985$ \\
$\mathrm{pH}$ & -7.892 & 3.078 & 6.573 & 0.010 & $<0.001$ & $0.000-0.156$ \\
\hline
\end{tabular}




\section{Discussion}

Previous studies have reported a wide range of BPD prevalence from $11 \%$ to $50 \%$, likely because of differences in gestational age or birth weight criteria for confirmed a BPD diagnosis [5-10]. A multicenter retrospective analysis from China showed that the incidence of BPD decreases with increase in gestational age and weight [11], which is consistent with the results of previous studies. The incidence of BPD in premature infants enrolled in this study was $38 \%$, which was significantly higher than that reported in other studies [11-12]. However, given the limitation of enrollment conditions, many premature infants who were not enrolled did not stay in hospital for 28 days, and all premature infants enrolled in this study were aged $<32$ gestational weeks, which reduced overall cohort size. As our hospital's NICU is a neonatal critical care center, all patients admitted here have serious health conditions. Understandably, the actual incidence of BPD in our hospital's NICU is lower than the statistical results of this study.

Gestational age is a known risk factor for BPD, and the incidence of BPD increases with the decrease of gestational age [2]. Lung development in premature infants, especially in extremely preterm infants, is often interrupted in the vesicle stage. This means that in addition to inadequate number of pulmonary alveoli, the alveolar function is single and the gas 
exchange, insufficient. Inflammatory response is often induced after birth and inflammatory mediators are activated due to disease changes in various systems, leading to the occurrence of BPD [13-14]. Univariate analysis showed that the use rates of invasive and non-invasive mechanical ventilation in the BPD group were higher than those in the control group, and the duration of non-invasive mechanical ventilation was also significantly higher than that in the control group. However, in the regression analysis, only the use rate of invasive mechanical ventilation was a risk factor for BPD. This was related to the relatively severe condition of premature infants in the BPD group after birth. Multi-center studies and meta-analysis $[11,15]$ have suggested that the extension of mechanical ventilation time may increase the risk of ventilator-associated pneumonia and cause ventilator-associated lung injury such as barometric injury and oxygen toxicity, further leading to impaired alveolar function and pulmonary fibrosis, eventually resulting in BPD. However, our regression analysis showed that neither invasive nor non-invasive ventilation duration was a high risk factor in either group, which may be related to the improvement of clinical rescue technology and airway management. Lung-protective ventilation strategy was mostly adopted in critically ill premature infants. Therefore, clinicians should carefully choose invasive or non-invasive ventilation based on the actual requirement and should not delay treatment to or overtreat patients owing 
to excessive concern about the occurrence of BPD.

Braldi et al. [16] showed that the use of blood products was a high risk factor for BPD. In this study, the use of plasma, erythrocyte suspension, and propyl spheres in premature infants in the BPD group was higher than the use in control group; however, regression analysis showed that only use of erythrocyte suspension was a high risk factor for BPD, which was consistent with previously reported results [17]. Excessive production of ferrous ions after infusion-induced erythrocyte destruction promotes the production of oxygen radicals and increases heme concentration, leading to overactivation of heme oxygenase, thereby aggravating lung injury. Demirel et al. [18] suggested that the infusion volume of erythrocyte suspension was more critical, but we found no statistically significant difference in the infusion volume of blood products between the two groups in our study. Premature infants in the BPD group were sicker than those in the control group, and the proportion and number of blood products used were also higher than those in the control group. These results need to be further verified in a large, double-blind, randomized controlled trial.

The results of our study showed that in the first week after birth, fluid restriction did not reduce the incidence of BPD, but premature infants had abnormal pulmonary fluid balance and poor liquid tolerance. Even normal fluid intake could lead to pulmonary interstitial and alveolar 
edema, causing further deterioration of pulmonary function. Therefore, fluid intake should be strictly controlled [19]. Previous studies had suggested that insufficient calorie intake and excessive fluid intake in early postnatal period were high risk factors for BPD [20-21]. Our results suggest that fluid intake, urine output, and calorie intake of premature infants in the control group were significantly higher than those in the BPD group in the first week after birth. Premature infants in the BPD group suffered from severe pulmonary disease in the early stage, many systemic complications, and limited fluid intake. These preterm infants had a younger gestational age, lower body weight, slower increase in oral feeding, and inadequate calorie intake in the early postpartum period. These factors also resulted in decreased lung development after birth and decreased ability to repair lung injury.

Continuous left-to-right shunt during PDA opening can lead to increased pulmonary blood flow and pulmonary fluid, increased pulmonary vascular injury and pulmonary hypertension, increased right ventricular load, and decreased lung function, which may increase the risk and severity of BPD. The results of this study showed that the incidence of PDA in the BPD group was significantly higher than that in the control group; however, consistent with previous results [2], PDA was not considered an independent risk factor for BPD in the regression analysis. Mohamed's study [22] suggested that the incidence of PDA in preterm 
infants aged $<28$ gestational weeks could be as high as $70 \%$, but conservative treatment did not increase mortality. Whether PDA promotes BPD is still controversial.

Previous studies suggested that the pathological mechanism leading to BPD was injury to the immature lung and abnormal repair of lung tissue after injury caused by adverse factors such as oxygen poisoning, barometric injury, volume injury, infection, or inflammation [19, 23]. However, our data showed that gestational age, invasive mechanical ventilation, use of erythrocyte suspension, insufficient calorie intake, low $\mathrm{pH}$ value, $\mathrm{PO} 2$ decrease, and $\mathrm{PCO} 2$ increase in the early postnatal blood gas analysis were the risk factors for BPD. To the best of our knowledge, there are no reports yet on the effects of $\mathrm{pH}, \mathrm{PO} 2$, and $\mathrm{PCO} 2$ on $\mathrm{BPD}$ in the early postnatal blood gas analysis. After 25 weeks of gestation, the original alveoli appear, and the distal bronchus continues to lengthen and expand, eventually forming the terminal vesicle, followed by the gradual appearance of the primitive and immature alveoli and the increase of alveolar volume and surface area. The fastest increase in the number of alveoli occurs at 32-38 weeks of gestation, and the alveoli gradually develop into mature alveoli after birth. Therefore, the intrauterine and postnatal environment of preterm infants aged $<32$ gestational weeks can affect lung development after birth, especially during rapid alveolar development $[13,19]$. During lung development, even mechanical 
ventilation with appropriate tidal volume and low oxygen concentration will affect the process of alveolarization and alveolar vascular development in premature infants, although the specific mechanism is not yet clear. The results of this study suggest that the $\mathrm{pH}$ value of the BPD group was lower than that of the control group in the blood gas analysis on the first day after birth. This index was an independent risk factor for BPD in the regression analysis. This may be related to the distribution of several $\mathrm{pH}$-sensitive cells on the ventral side of the medulla oblongata that are sensitive to subtle changes in the $\mathrm{pH}$ value of the local arterial blood within a certain range and project information to the respiratory center, thereby regulating respiration. At the same time, whether early $\mathrm{pH}$ value has an effect on alveolar development in premature infants, especially extremely premature infants, still needs further verification. In addition, the results of blood gas analysis in the first week after birth also indicated that $\mathrm{PCO} 2$ in the BPD group was higher than that in the control group on days 3 and 7 after birth, while PO2 in the BPD group was lower than that in the control group. In the early postnatal stage, owing to improved diagnosis and treatment and implementation of positive ventilation strategy, correction of hypoxemia and stability of internal environment in premature infants can be guaranteed. However, with the gradual development of alveoli after delivery, the alveolar function of premature infants in the control group was significantly better than that in 
the BPD group. Therefore, PCO2 in the BPD group was increased and PO2 was decreased in the blood gas analysis. Therefore, we speculated that the development of alveoli was greatly influenced by the internal environment; specifically, the internal environment in the first week after birth could predict the incidence of BPD. This also means that it is necessary to monitor and stabilize the internal environment in a timely manner to reduce the occurrence of BPD, but the specific mechanism involved remains to be better understood.

The occurrence of BPD is the result of several factors. Improving maternal-fetal medicine, avoiding premature delivery, maintaining stability of the early internal environment, increasing early energy intake after delivery, and reducing the infusion of erythrocyte suspension may be important means to reduce the incidence of BPD. The results of our study suggest that changes in $\mathrm{pH}, \mathrm{PCO} 2$, and $\mathrm{PO} 2$ levels in blood gas in the first week after birth may be one of the indicators for predicting BPD. However, this study is a single-center retrospective study, which may have biased the results to some extent. Therefore, multi-center, prospective studies with a larger sample size are required to improve clinical understanding and application.

\section{List of abbreviations}

BPD: Bronchopulmonary dysplasia; VLBW: very low birth weight; 
ELBW: extremely low birth weight; NICU: the neonatal intensive care unit; RDS: respiratory distress syndrome; PDA: patent ductus arteriosus

\section{Declarations}

\section{Ethics approval and consent to participate}

Ethical clearance was obtained from the Institutional Review Committee of the First Affiliated Hospital of Nanjing Medical University. Parents of each participant received an explanation about the purpose of study, and verbal informed consent was obtained from each participant prior to proceeding. The ethical committee formally waived the need of formal written consent since the study was done by retrospective analysis of patients who had been discharged from hospital. To ensure confidentiality, all collected data were coded and locked in a separate room prior to the data entry process. After entering of data into the computer, all data were locked by password. Participant names were not included in the data collection format, and the data were not disclosed to any person other than principal investigators.

\section{Consent for publication}

Not applicable. 
The data sets used and/or analyzed during the current study are available from the corresponding author on reasonable request.

\section{Competing interests}

The authors declare that they have no competing interests.

\section{Funding}

Not applicable.

\section{Authors' contributions}

Yue Li: Literature review, data analysis, interpretation and drafting the manuscript. Xiao Lin Miao: Data analysis and interpretation. Shu Dong Cui: Conception of research protocol, study design and manuscript review. All authors have read and approved the manuscript.

\section{Acknowledgements}

Not applicable.

\section{References:}

[1] Reiterer F, Scheuchenegger A, Resch B, Maurer-Fellbaum U, Avian A,

Urlesberger B. Bronchopulmonary dysplasia in very preterm infants:

Outcome up to preschool age, in a single center of Austria. Pediatr Int. 
2019;61(4):381-7.

[2] Thébaud B, Goss KN, Laughon M, Whitsett JA, Abman SH,

Steinhorn RH, et al. Bronchopulmonary dysplasia. Nat Rev Dis Primers. 2019;5(1):78.

[3] Kolacz J, Porges SW. Corrigendum: chronic diffuse pain and functional gastrointestinal disorders after traumatic stress:

pathophysiology through a polyvagal perspective. Front Med (Lausanne). 2018;5:229.

[4] Jobe AH, Bancalari E. Bronchopulmonary dysplasia. Am J Respir Crit Care Med. 2001;163(7):1723-9.

[5] Adams M, Bassler D, Bucher HU, Roth-Kleiner M, Berger TM, Braun $\mathrm{J}$, et al. Variability of very low birth weight infant outcome and practice in Swiss and US neonatal units. Pediatrics. 2018;141(5). pii: e2017343 [6] Su BH, Hsieh WS, Hsu CH, Chang JH, Lien R, Lin CH, et al. Neonatal outcomes of extremely preterm infants from Taiwan: comparison with Canada, Japan, and the USA. Pediatr Neonatol. 2015;56(1):46-52.

[7] Lin HJ, Du LZ, Ma XL, Shi LP, Pan JH, Tong XM, et al. Mortality and morbidity of extremely low birth weight infants in the mainland of China: a multi-center study. Chin Med J (Engl). 2015;128(20):2743-50. [8] Bhunwal S, Mukhopadhyay K, Bhattacharya S, Dey P, Dhaliwal LK. Bronchopulmonary dysplasia in preterm neonates in a level III neonatal 
unit in India [J]. Indian Pediatr. 2018;55(3):211-5.

[9] Lee JH, Noh OK, Chang YS; Korean Neonatal Network. Neonatal outcomes of very low birth weight infants in Korean Neonatal Network from 2013 to 2016. J Korean Med Sci. 2019;34(5):e40.

[10] Isayama T, Lee SK, Yang J, Lee D, Daspal S, Dunn M, et al. Revisiting the definition of bronchopulmonary dysplasia: Effect of changing panoply of respiratory support for preterm neonates. JAMA Pediatr. 2017;171(3):271-9.

[11] Collaborative Study Group for Bronchopulmonary Dysplasia of Prematurity in China. Incidence and risk factors of bronchopulmonary dysplasia in premature infants in 10 hospitals in China. Zhonghua Er Ke Za Zhi. 2011;49(9):655-62.

[12] Jensen EA, Schmidt B. Epidemiology of bronchopulmonary dysplasia. Birth Defects Res A Clin Mol Teratol. 2014;100(3):145-57. [13] Baker CD, Abman SH, Mourani PM. Pulmonary hypertension in preterm infants with bronchopulmonary dysplasia. Pediatr Allergy Immunol Pulmonol. 2014;27(1):8-16.

[14] Shahzad T, Radajewski S, Chao CM, Bellusci S, Ehrhardt H. Pathogenesis of bronchopulmonary dysplasia: when inflammation meets organ development. Mol Cell Pediatr. 2016;3(1):23.

[15] Isayama T, Iwami H, McDonald S, Beyene J. Association of noninvasive ventilation strategies with mortality and bronchopulmonary 
dysplasia among preterm infants: A systematic review and meta-analysis. JAMA. 2016;316(6):611-24.

[16] Baraldi E, Filippone M. Chronic lung disease after premature birth. N Engl J Med. 2007;357(19):1946-55.

[17] Ghirardello S, Dusi E, Cortinovis I, Villa S, Fumagalli M, Agosti M, et al. Effects of red blood cell transfusions on the risk of developing complications or death: an observational study of a cohort of very low birth weight infants. Am J Perinatol. 2017;34(1):88-95.

[18] Demirel N, Bas AY, Zenciroglu A. Bronchopulmonary dysplasia in very low birth weight infants. Indian J Pediatr. 2009;76(7):695-8.

[19] Shao XM, Ye HM, Qiu XS. Practice of neonatology. 4th ed. Beijing: People's medical publishing house; 2011.

[20] Oh W, Poindexter BB, Perritt R, Lemons JA, Bauer CR, Ehrenkranz RA, et al. Association between fluid intake and weight 1oss during the first ten days of life and risk of bronchopulmonary dysplasia in extremely low birth weight infants. J Pediatr. 2005;147(6):786-90.

[21] Maayan-Metzger A, Mazkereth R, Kuint J. Weight loss and bronchopulmonary dysplasia in very low birth weight infants. Fetal Pediatr Pathol. 2008;27(4-5):215-22.

[22] Mohamed MA, El-Dib M, Alqahtani S, Alyami K, Ibrahim AN, Aly H. Patent ductus arteriosus in premature infants: to treat or not to treat? J Perinatol. 2017;37(6):652-7. 
[23] Collins JJP, Tibboel D, de Kleer IM, Reiss IKM, Rottier RJ. The Future of Bronchopulmonary Dysplasia: Emerging Pathophysiological Concepts and Potential New Avenues of Treatment. Front Med (Lausanne). 2017;4:61. 